\title{
Phase resolved X-ray spectroscopy of HDE 228766: Probing the wind of an extreme $\mathrm{Of}^{+} /$WNLha star ${ }^{\star}$ (Corrigendum)
}

\author{
G. Rauw ${ }^{1}$, L. Mahy ${ }^{1}$, Y. Nazé ${ }^{1, \star \star}$, P. Eenens ${ }^{2}$, J. Manfroid ${ }^{1, \star \star \star}$, and C. A. Flores ${ }^{2}$ \\ ${ }^{1}$ Groupe d'Astrophysique des Hautes Energies, Institut d'Astrophysique et de Géophysique, Université de Liège, Allée du 6 Août, \\ Bât B5c, 4000 Liège, Belgium \\ e-mail: rauw@astro.ulg.ac.be \\ 2 Departamento de Astronomia, Universidad de Guanajuato, Apartado 144, 36000 Guanajuato, GTO, Mexico
}

A\&A 566, A107 (2014), DOI: 10.1051/0004-6361/201423885

Key words. binaries: spectroscopic - stars: early-type - stars: mass-loss - stars: winds, outflows - X-rays: stars - errata, addenda

In the title of the paper by Rauw et al. (2014), the HDE number of the target was wrongly quoted as HDE 288766, instead of HDE 228766.

\section{References}

Rauw, G., Mahy, L., Nazé, Y., et al. 2014, A\&A, 566, A107

\footnotetext{
* Based on observations collected with XMM-Newton, an ESA science mission with instruments and contributions directly funded by ESA member states and the USA (NASA), and on data collected at the San Pedro Mártir observatory (Mexico).

$\star \star$ Research Associate FRS-FNRS (Belgium).

$\star \star \star$ Honorary Research Director FRS-FNRS (Belgium).
} 